\title{
A New Optimal Fuzzy Logic Guidance Law using Time Variant Particle Swarm Optimization
}

\author{
Labeed Hassan \\ Department of Aerospace Engineering \\ Malek Ashtar University \\ Islamic Republic of Iran
}

\author{
Seyed Hossein Sadati \\ Space Research Institute \\ Islamic Republic of Iran
}

\author{
Mohamad Bagher Malaeak \\ Department of Aerospace Engineering \\ Sharif University \\ Islamic Republic of Iran
}

\author{
Mohamad Ali Ashtiani \\ Department of Aerospace Engineering \\ Malek Ashtar University \\ Islamic Republic of Iran
}

\author{
Jalal Karimi \\ Department of Aerospace Engineering \\ Malek Ashtar University \\ Islamic Republic of Iran
}

\begin{abstract}
New Optimal Fuzzy Logic Guidance (NOFLG) law for the class of surface to air homing missile is proposed. The introduced approach is a modification of Optimal Fuzzy Logic Guidance (OFLG) law. Time Variant Particle Swarm Optimization (TVPSO) is used to optimize both Membership Functions (MFs) and rules' weights of the proposed design. The performance of the new guidance law is compared with that of OFLG one. Different case-studies show that the current approach provides better performance in regard to; miss distance, flight time and control effort.
\end{abstract}

\section{Keywords}

Fuzzy Logic, Time Variant Particle Swarm Optimization, Proportional Navigation Guidance.

\section{INTRODUCTION}

Most guidance laws used in current missiles are mainly based on classical control techniques $[1,2]$. These techniques, although they are efficient in many cases, might not be effective for tracking and interception of maneuvering targets. In addition, there are a perfect knowledge of system requirement and a large computational one. Fuzzy Logic Controllers (FLCs) have number of suitable properties that diminish such difficulties, therefore, they have been used in many fields where plants exhibit uncertainties or we lack a rigorous model to describe their behavior. On the other hand, most fuzzy guidance laws are fuzzy-logic implementation of existing well-known classical guidance laws; such as, Proportional Navigation Guidance (PNG) that is because of its simplicity, effectiveness and ease of implementation [3].

In fact, tuning the parameters of FLCs is time consuming and frustrating exercise. Particle Swarm Optimization (PSO) is considered suitable technique to overcome these difficulties because of its simplicity. PSO which is first developed by Kennedy and Eberhart [4] is a population based stochastic optimization technique was inspired by social behavior patterns of organisms that live and interact within large groups as flocks of birds, schools of fish, or swarms of bees. It is one of the modern heuristic algorithms that was developed through simulation of a simplified social system, and has been found to be robust in solving complex optimization system. In the current study, a new version of PSO that so-called Time Variant Particle Swarm Optimization TVPSP [5] will be used to investigate the NOFLG law. The investigated design will be compared with OFLG that had been introduced in our previous study [6].

The paper is organized as follows: an overview on the preintroduced OFLG law is briefed in Section 2. The basic PSO and TVPSO are explained in Section 3. The optimization process is discussed in Section 4. In Section 5 results and analysis are provided. Conclusions are then provided in Section 6.

\section{BRIEF OVERVIEW ON OFLG LAW}

The pre-introduced OFLG has been built upon the conception of PNG law. The PNG law gives commanded acceleration $A_{c}$, on its output, proportional to its two inputs; $\dot{\lambda}$ (angle rate of the instantaneous line-of-sight) and $V_{c}$ (target-missile closing velocity). Mathematically it can be expressed as:

$$
A_{c}=N V_{c} \dot{\lambda}
$$

Where; $N$ is navigation constant defined by the designer. In our previous study, the OFLG has 49 rules with optimized triangular MFs and non-optimized weights. Each rule has weight equals 1. The rules were tabulated as plotted in Figure 1:

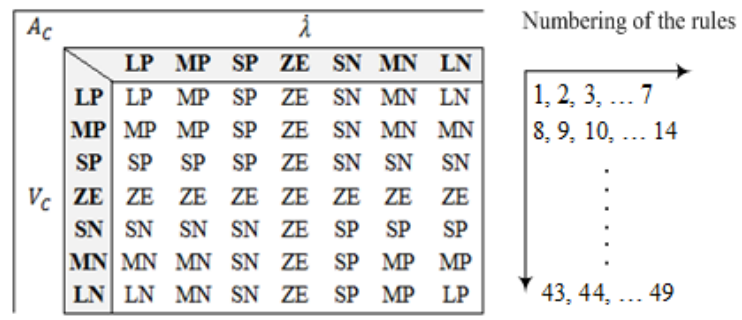

Fig. 1: Entire Rules of OFLG

In the current work, the same rules will be used. The parameters of the MFs and the weights of the rules will be optimized using TVPSO.

\section{TVPSO}

In the PSO every individual $i$ of the swarm contains parameters for position $x_{i}$ and velocity $v_{i}$. The new position 
of each particle is calculated by adding a velocity to the current position.

In particular, the velocity associated with each particle is calculated as [7]:

$$
\begin{array}{r}
v_{i}(k+1)=w v_{i}(k)+c_{1} \cdot r_{1}(k)\left(x_{i}^{g}-x_{i}(k)\right)+ \\
c_{2} \cdot r_{2}(k)\left(x_{i}^{p}-x_{i}(k)\right)
\end{array}
$$

Where;

$w$ - Inertia weight factor.

$v_{i}(k)$-Velocity of the particle $i$ at time step $k$.

$x_{i}^{g}$ - Global best performing particle up to time step $k$.

$x_{i}^{p}$ - Personal best performing particle up to time step $k$.

$x_{i}(k)$-Current location of particle $i$.

$c_{2}, c_{2}$ - Constants usually equal each to other.

$r_{1}, r_{2}$ - Random numbers within $[0,1]$ those represent random fiction.

To limit the searching space $v_{i}(k+1)$ is limited to be within a certain range of $v_{\text {imin }} \leq v_{i} \leq v_{\text {imax }}$. The new location of the particle $i$ can be calculated as:

$x_{i}(k+1)=x_{i}(k)+v_{i}(k+1)$

(3)

The following Figure illustrates how updating of the particle position is achieved according to the PSO's Equations.

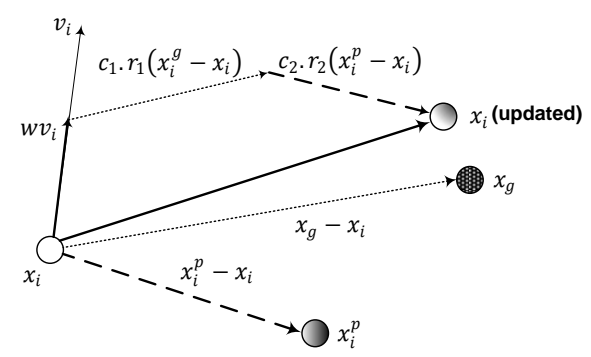

Fig. 2: Visualizing PSO.

In the TVPSO, the vital parameters; inertia weight $w$ and acceleration coefficients $c_{1}, c_{2}$, are allowed to be changed with iterations it, making it capable of effectively handling optimization problems of different characteristics. Here, the parameters; $w, c_{1}$ and $c_{2}$ are renamed as; $w_{i t}, c_{1 i t}$ and $c_{2 i t}$ respectively.

The inertia weight $w_{i t}$ controls the influence of the current velocity on the new velocity. A large inertia weight compels large exploration through the search space; a smaller inertia weight causes reduced exploration. The value of $w_{i t}$ is allowed to decrease linearly with iteration from $w_{\max }$ to $w_{\min }$ as:

$w_{i t}=\left(w_{\max }-w_{\min }\right) \frac{I-i t}{i t}+w_{\min }$

(4)

Where; $I$ - Maximum number of iterations.

The parameters $c_{1 i t}$ and $c_{2 i t}$, are called cognitive acceleration coefficient and social acceleration coefficient respectively. The acceleration limits the trajectory of the particle oscillation. The smaller the acceleration, the smoother the trajectory of the particle is. However, too small acceleration may lead to slow convergence, whereas too large acceleration drives the particles towards infinity.
To incorporate better compromise between the exploration and exploitation of the search space in the swarm, $c_{1 i t}$ has been allowed to decrease from its initial value of $c_{1 \max }$ to $c_{1 \min }$ while $c_{2 i t}$ has been increased from $c_{2 \max }$ to $c_{2 \min }$ as follows:

$c_{1 i t}=\left(c_{1 \max }-c_{1 \min }\right) \frac{i t}{I}+c_{1 \max }$

(5)

$c_{2 i t}=\left(c_{2 \max }-c_{2 \min }\right) \frac{i t}{I}+c_{2 \max }$

(6)

The flowchart of the TVPSO is shown in as the following:

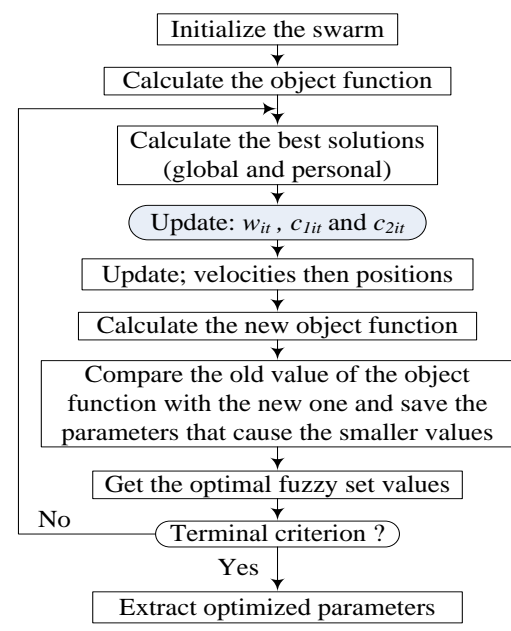

Fig. 3: Flowchart of TVPSO.

The swarm is initialized randomly and the object function is calculated. The algorithm updates the particle's velocity and adds the new velocity to the old position to calculate the new position. The updating of the velocity is influenced by both $x_{i}^{g}$, the best global solution associated with the lowest cost (objective function) ever found by a particle, and $x_{i}^{p}$, the best local solution associated with the lowest cost in the present population. The solution $x_{i}^{p}$ is replaced with $x_{i}^{g}$ when it causes less cost than the $x_{i}^{g}$ does. The algorithm continues updating the velocities and adds them to the corresponding positions to evaluate the objective function until a termination criterion, such as a limit on the number of iterations or satisfactory results, is reached, thereupon the process is stopped.

\section{OPTIMIZATION PROCESS}

The population is set to be $P=100$ particles and the total searching iteration is set to be $I=500$. As suggested in [5] the following values are used throughout the optimization process:

$w_{\max }=0.7, w_{\min }=0.4, c_{1 \max }=c_{2 \max }=2.5, c_{1 \min }=$

$c_{2 \min }=0.5$. The object function $F\left(A_{\text {par }}\right)$ is defined as follows:

$F\left(A_{\text {par }}\right)=k_{1} \cdot \int_{0}^{t_{f}} A_{C}^{2} d t+k_{2} \cdot R_{T M}\left(F_{t}\right)+k_{3} F_{t}$

(7)

The first term denotes the control effort $C_{E f f}$ while the last two ones denote the miss distance $M_{D}$ and flight time $F_{t}$. Where: $A_{\text {par }}$ is an array that includes the parameters needed 
to be optimized, $R_{T M}$ is the target-missile relative distance, whilst $k_{1}, k_{2}$ and $k_{3}$ are constants those referring to the terms' preference. In the current study, it supposed that all the terms have same importance, so that $k_{1}=1 / C_{E f f_{\max }}, k_{2}=$ $1 / R_{T M\left(F_{t}\right)_{\max }}$, and $k_{3}=1 / F_{t_{\max }}$, where $C_{E f f_{\max }}, R_{T M\left(F_{t}\right)_{\max }}$ and $F_{t_{\max }}$ are the maximum allowable values of the terms defined by the designer regard to the missile's ability.

The flowing figure illustrates the overall process which is built using MatLab environment:

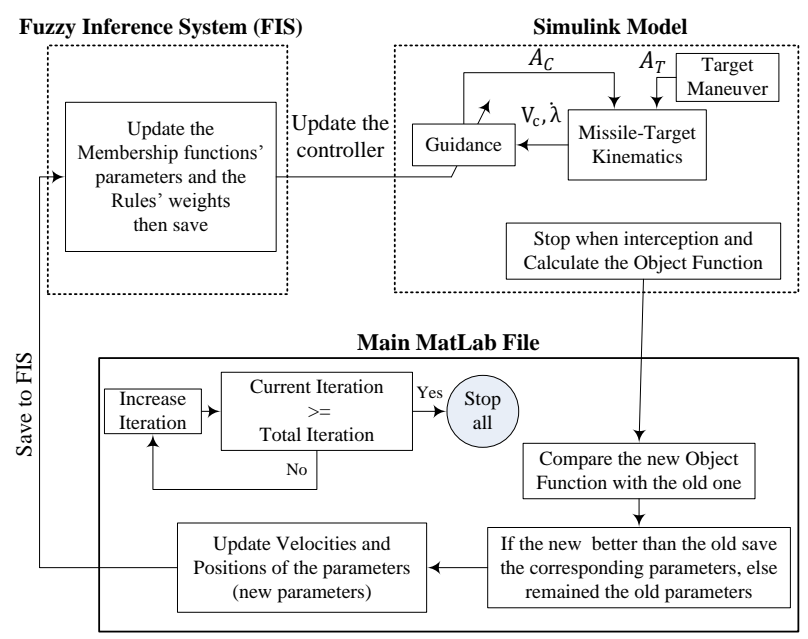

Fig. 4: The flow chart of overall process.

During the optimization process, the value's decrement of the object function can be noted as shown in Figure 5. Also it can be seen that, the searching can be terminated after about 400 iterations when there is no reduction was observed.

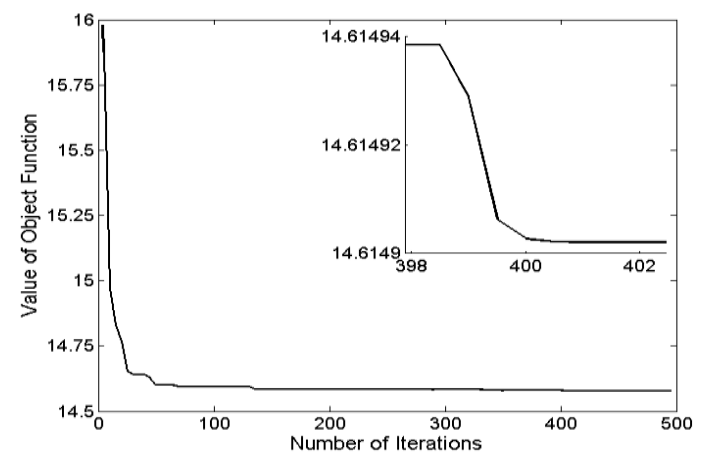

Fig. 5: The value of the object function.

As soon as the process is completed, the optimized parameters of the inputs $\dot{\lambda}, V_{C}$ and the output $A_{C}$ can be extracted. The optimized parameters of the MFs and the rules' weights are plotted as following:

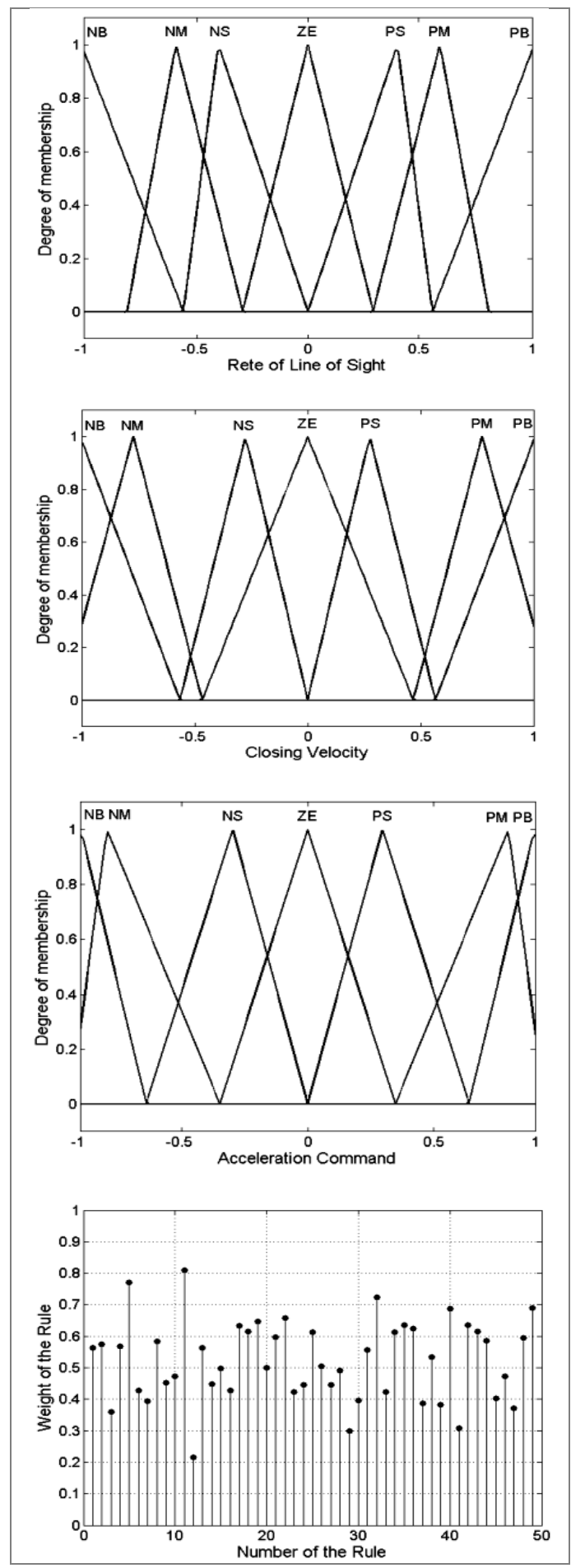

Fig. 6: The Optimized parameters of the NOFLG.

\section{RESULTS AND ANALYSIS}

During the simulation we suppose the missile and the target are located at $(0,0) \mathrm{km}$ and $(10,3) \mathrm{km}$ and moves with velocities $1000 \mathrm{~m} / \mathrm{sec}$ and $300 \mathrm{~m} / \mathrm{sec}$ respectively. Also we considered the maximum target ability to maneuver within $[-3$ up to 6$] \mathrm{g}$, where $g=9.81$ is the gravity constant. Finally the missile has a dynamic saturation within $[-15$ up to 15$] \mathrm{g}$.

The simulation is achieved for 20 scenarios with respect to different target's maneuvers. The examined scenarios are 
suggested when the values of $A_{T}=[-3,-2.5, \ldots 5.5,6] g$. Root Mean Square value (RMS) of: $M_{D}, F_{t}$ and $C_{E f f}$ are calculated and listed in Table 1 .

Table 1: calculated RMS of the values.

\begin{tabular}{lrlc}
\hline RMS & $\boldsymbol{M}_{\boldsymbol{D}}[\mathbf{m}]$ & $\boldsymbol{F}_{\boldsymbol{t}}[\mathbf{s e c}]$ & $\boldsymbol{C}_{\boldsymbol{E f f} \boldsymbol{f}}\left[\mathbf{m}^{\mathbf{2}} / \mathbf{s e c}^{\mathbf{3}}\right] \times \mathbf{1 0}^{-\mathbf{4}}$ \\
\hline OFGL & 5.71 & 8.21 & 4.52 \\
NOFLG & 5.24 & 8.11 & 4.06 \\
\hline
\end{tabular}

It is clear that the NOFLG law overweighs OFLG law in all of the terms and insures $8 \%, 1 \%$ and $10 \%$ improvement to $M_{D}$, $F_{t}$ and $C_{E f f}$ respectively. In other words one can say that; TVPSO gives better improvement than PSO.

The following two figures show the trajectories and the accelerations for a difficult scenario that is when the target is considered to have its maximum ability with a square acceleration within $[-3,6] \mathrm{g}$.

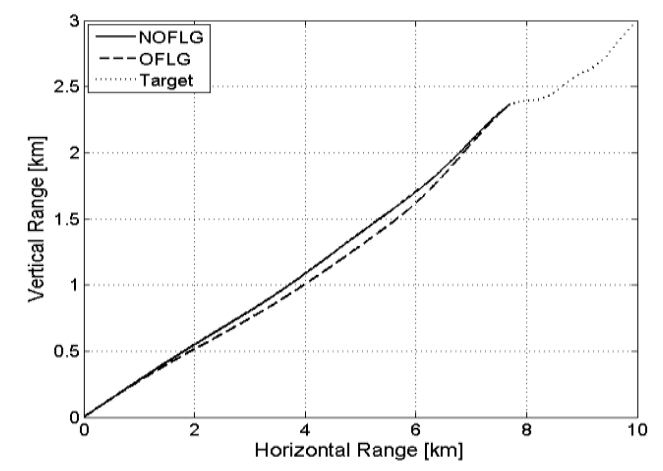

Fig. 7: Trajectories.

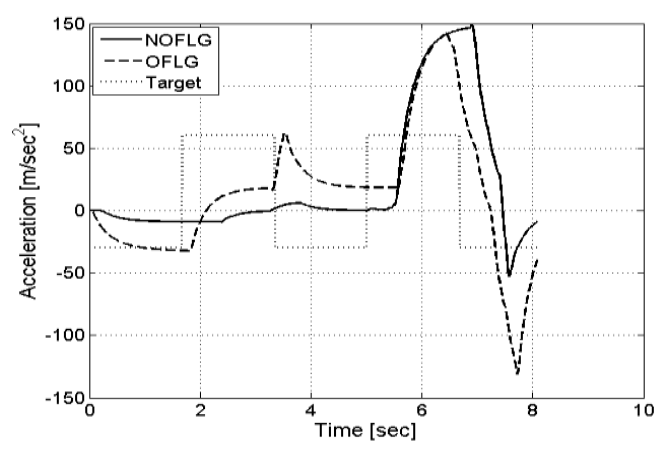

Fig. 8: Accelerations.
Figure 7 shows the trajectories achieved by the missile when using OFLG law and NOFLG law in addition to the target trajectory. While Figure 8 plots the changing of the corresponding acceleration commands.

\section{CONCLUSION}

In this work, a NOFLG law is investigated. Parameters of the MFs and rules' weights of the proposed design are optimized using TVPSO algorithm. The optimization is achieved under the consideration of minimizing the object function. Many cases where the target makes different maneuvering have been studied. The resulted NOFLG law is compared with an OFLG law. The results show that NOFLG behaves better in other words. The study verifies that the TVPSO performs better than the PSO.

\section{REFERENCES:}

[1] A. Moharampour, J. Poshtan and A. Khaki Sedigh, 2010. "A Modified Proportional Navigation Guidance for Accurate Target Hitting." Iranian Journal of Electrical \& Electronic Engineering, Vol. 6, No. 1, pp 20-28.

[2] Z. Shiyu, Z. Rui, W. Chen, and D. Quanxin, 2010. "Design of Time-constrained Guidance Laws via Virtual Leader Approach." Chinese Journal of Aeronautics, Vol. 23,No. 1 pp.103-108.

[3] P. Zarchan, 2007. "Tactical and Strategic Missile Guidance," $5^{\text {th }}$ edition, AIAA,

[4] Clerc, M. and Kennedy, J. "The particle swarm explosion, stability, and convergence in a multidimensional complex space". 2002. IEEE Transactions on Evolutionary Computation, Vol 6, No. 1, pp. 58-73.

[5] Praveen Kumar Tripathi, Sanghamitra Bandyopadhyay and Sankar Kumar Pal, 2007. "Multi Objective Particle Swarm Optimization with time variant inertia and acceleration coefficients." Information Sciences Vol. 177, No. 22, pp. 5033-5049.

[6] Labeed Hassan, Seyed Hossein Sadati and Jalal Karimi, 2013. "An Optimal Fuzzy Logic Guidance Law Using Particle Swarm Optimization.” International Journal of Computer Applications, Vol. 69, No. 3, pp. 40-47.

[7] Qinghai Bai, 2010. "Analysis of Particle Swarm Optimization Algorithm." CCSE, computer and information science, Vol. 3, No. 1. PP. 180-184. 